PROCEEDINGS OF THE

AMERICAN MATHEMATICAL SOCIETY

Volume 127, Number 5, Pages 1409-1411

S 0002-9939(99)04697-3

Article electronically published on January 29, 1999

\title{
THE MAXIMAL IDEAL SPACE OF $H^{\infty}(\mathbb{D})$ WITH RESPECT TO THE HADAMARD PRODUCT
}

\author{
HERMANN RENDER
}

(Communicated by Albert Baernstein II)

\begin{abstract}
It is shown that the space of all regular maximal ideals in the Banach algebra $H^{\infty}(\mathbb{D})$ with respect to the Hadamard product is isomorphic to $\mathbb{N}_{0}$. The multiplicative functionals are exactly the evaluations at the $n$-th Taylor coefficient. It is a consequence that for a given function $f(z)=\sum_{n=0}^{\infty} a_{n} z^{n}$ in $H^{\infty}(\mathbb{D})$ and for a function $F(z)$ holomorphic in a neighborhood $U$ of 0 with $F(0)=0$ and $a_{n} \in U$ for all $n \in \mathbb{N}_{0}$ the function $g(z)=\sum_{n=0}^{\infty} F\left(a_{n}\right) z^{n}$ is in $H^{\infty}(\mathbb{D})$.
\end{abstract}

\section{INTRODUCTION}

Let $\mathbb{D}:=\{z \in \mathbb{C}:|z|<1\}$ be the open unit disk and let $f(z)=\sum_{n=0}^{\infty} a_{n} z^{n}$ and $g(z)=\sum_{n=0}^{\infty} b_{n} z^{n}$ be power series on $\mathbb{D}$. Then the Hadamard product of $f$ and $g$ is defined by $f * g(z)=\sum_{n=0}^{\infty} a_{n} b_{n} z^{n}$. The Hadamard product on the space $H(\mathbb{D})$ of all holomorphic functions on $\mathbb{D}$ is continuous with respect to the topology of compact convergence. In [1] R. Brooks has shown that the space of all maximal ideals in the space $H(\mathbb{D})$ is isomorphic to the Stone-Čech-compactification $\beta \mathbb{N}_{0}$ of $\mathbb{N}_{0}:=\mathbb{N} \cup\{0\}$ and the multiplicative functionals on $H(\mathbb{D})$ are given by the coefficient functionals $\delta_{n}: H(\mathbb{D}) \rightarrow \mathbb{C}$ defined by $\delta_{n}(f):=a_{n}$ (where $f(z)=\sum_{n=0}^{\infty} a_{n} z^{n}$ in $|z|<1$ and $n \in \mathbb{N}_{0}$ ). In this note we discuss the subalgebra $H^{\infty}(\mathbb{D})$ of all bounded holomorphic functions which has been considered for example in [3]. Our main result states that the non-trivial multiplicative functionals on $H^{\infty}(\mathbb{D})$ are of the form $\delta_{n}, n \in \mathbb{N}_{0}$ (as in the case of $H(\mathbb{D}))$. In contrast to the algebra $H(\mathbb{D})$ the space $H^{\infty}(\mathbb{D})$ is even a Banach algebra with respect to the supremum norm which is denoted by $\|f\|_{\infty}$ for $f \in H^{\infty}(\mathbb{D})$. It follows that the maximal modular ideals of $H^{\infty}(\mathbb{D})$ are the kernels of the multiplicative functionals and therefore the space of all maximal modular ideals of $H^{\infty}(\mathbb{D})$ is isomorphic to $\mathbb{N}_{0}$. Note that $H(\mathbb{D})$ possesses a unit element $\gamma(z)=\frac{1}{1-z}=\sum_{n=0}^{\infty} z^{n}$ which is not in the subalgebra $H^{\infty}(\mathbb{D})$.

\section{THE RESULTS}

Let $B$ be the space of all $f(z)=\sum_{n=0}^{\infty} a_{n} z^{n}$ such that $\sum_{n=0}^{\infty}\left|a_{n}\right|<\infty$; clearly, $\|f\|_{\infty} \leq \sum_{n=0}^{\infty}\left|a_{n}\right|$, so $B \subset H^{\infty}(\mathbb{D})$. We note that if $f=\sum_{n=0}^{\infty} a_{n} z^{n} \in H^{\infty}(\mathbb{D})$, then $\sum_{n=0}^{\infty}\left|a_{n}\right|^{2}=\|f\|_{2}^{2} \leq\|f\|_{\infty}^{2}<\infty$, where $\|f\|_{2}:=\sqrt{\sum_{n=0}^{\infty}\left|a_{n}\right|^{2}}$. Hence for any $f, g \in H^{\infty}(\mathbb{D})$, we have $f * g \in B$, since $\sum_{n=0}^{\infty}\left|a_{n} b_{n}\right| \leq\|f\|_{2}\|g\|_{2}$ by the

Received by the editors March 27, 1997 and, in revised form, August 19, 1997.

1991 Mathematics Subject Classification. Primary 46J15; Secondary $30 B 10$.

Key words and phrases. Hadamard product, bounded analytic functions. 
Cauchy- Schwarz inequality; this also shows that $H^{\infty}(\mathbb{D})$ is a Banach algebra under Hadamard multiplication.

Proposition 1. Let $A$ be the Banach algebra obtained by adjoining a unit to $H^{\infty}(\mathbb{D})$. If $f=\sum_{n=0}^{\infty} a_{n} z^{n} \in B$, then $\sigma_{A}(f)=\left\{a_{n}: n \in \mathbb{N}_{0}\right\} \cup\{0\}$.

Proof. We must show that if $\lambda \notin\left\{a_{n}: n \in \mathbb{N}_{0}\right\}$ and $\lambda \neq 0$, then $\lambda-f$ is invertible in $A$ (the other inclusion is easy). Let $g(z)=\sum_{n=0}^{\infty} \frac{a_{n}}{\lambda-a_{n}} z^{n}$; since $\left|a_{n}\right|<|\lambda| / 2$ for sufficiently large $n$, we have $\left|a_{n} /\left(\lambda-a_{n}\right)\right| \leq(2 /|\lambda|)\left|a_{n}\right|$ for sufficiently large $n$, so $g \in B \subset H^{\infty}(\mathbb{D})$. Since

$$
\lambda g-f=\sum_{n=0}^{\infty} \frac{\lambda a_{n}-\lambda a_{n}+a_{n}^{2}}{\lambda-a_{n}} z^{n}=f * g,
$$

we see that $(\lambda-f) *(1+g)=\lambda$, so $\lambda-f$ is invertible in $A$.

The next result is the main step of our proof. Although we need it only for Banach algebras, it is valid for the larger class of all Fréchet algebras, cf. [4] for definition. We denote by $\Delta_{A}$ the set of all continuous multiplicative non-trivial functionals.

Theorem 2. If $A$ is a unital Frechet algebra, and $S$ is a countable subset of $\Delta_{A}$ with the property that $\sigma_{A}\left(f^{2}\right)=\left\{\varphi\left(f^{2}\right): \varphi \in S\right\}$ for all $f \in A$, then $S=\Delta_{A}$.

Proof. Let $S=\left\{\varphi_{n}: n \in \mathbb{N}\right\}$. Suppose that there exists $\varphi \in \Delta_{A} \backslash S$. As $\varphi \neq \varphi_{n}$ for all $n \in \mathbb{N}$, the sets $A_{n}:=\operatorname{ker}\left(\varphi_{n}-\varphi\right)$ and $B_{n}:=\operatorname{ker}\left(\varphi_{n}+\varphi\right)$ are closed hyperplanes, in particular they are nowhere dense. By the Baire category theorem there exists $f \in A$ such that $f \notin A_{n}$ and $f \notin B_{n}$ for all $n \in \mathbb{N}$. This means that $\varphi_{n}(f) \neq \varphi(f)$ and $\varphi_{n}(f) \neq-\varphi(f)$ for all $n \in \mathbb{N}$. On the other hand we know that $\lambda:=\varphi(f) \in \sigma_{A}(f)$ since $\varphi$ is multiplicative. Hence $\lambda^{2} \in \sigma_{A}\left(f^{2}\right)$. By assumption there exists $n \in \mathbb{N}$ with $\lambda^{2}=\varphi_{n}\left(f^{2}\right)=\left(\varphi_{n}(f)\right)^{2}$. Hence $\lambda=\varphi_{n}(f)$ or $\lambda=-\varphi_{n}(f)$, a contradiction.

Theorem 3. The non-trivial multiplicative functionals on $H^{\infty}(\mathbb{D})$ are of the form $\delta_{n}, n \in \mathbb{N}_{0}$.

Proof. By the above, $f^{2} \in B$ for all $f \in H^{\infty}(\mathbb{D})$. Now apply Proposition 1 and Theorem 2.

Theorem 4. Let $U$ be an open neighborhood of zero and $F: U \rightarrow \mathbb{C}$ holomorphic with $F(0)=0$. If $f(z)=\sum_{n=0}^{\infty} a_{n} z^{n}$ is in $H^{\infty}(\mathbb{D})$ and $a_{n} \in U$ for all $n \in \mathbb{N}_{0}$, then $F(f)(z):=\sum_{n=0}^{\infty} F\left(a_{n}\right) z^{n}$ is in $H^{\infty}(\mathbb{D})$.

Proof. This is just the functional calculus for Banach algebras (without unit element) using the fact that $\sigma_{A}(f) \subset U$ by Theorem 3 .

Remark 5. There is no continuous functional calculus on $H^{\infty}(\mathbb{D})$. Consider for example $F(x)=|x|$. Let $g(z)=(1-z)^{-i}=\sum_{n=0}^{\infty} b_{n} z^{n}$. Then $F(g)=\sum_{n=0}^{\infty}\left|b_{n}\right| z^{n}$ is not bounded since $\left|b_{n}\right| \geq \frac{1}{n}$ and $\sum_{n=0}^{\infty}\left|b_{n}\right|$ is divergent; cf. [5, p. 68].

One should observe that analyticity plays no role, other than in the proof that $H^{\infty}(\mathbb{D})$ is a Banach algebra under Hadamard multiplication; since $H^{\infty}(\mathbb{D})$ is isometrically imbedded in $L^{\infty}(\mathbb{T})$, and the Hadamard product is just convolution, one can just as easily state and prove the corresponding theorem for $L^{\infty}(\mathbb{T})$, or any of 
its subspaces having the form $E=\left\{f \in L^{\infty}(\mathbb{T}): \hat{f}_{n}=0\right.$ for all $\left.n \notin S\right\}$, where $\hat{f}_{n}$ is the $n$th Fourier coefficient of $f$, and $S$ is any subset of $\mathbb{Z}$. Of course, $H^{\infty}(\mathbb{D})$ is the special case of $S=\mathbb{N}_{0}$. Each such $E$ is a Banach algebra under convolution, and every nontrivial homomorphism of $E$ to $\mathbb{C}$ has the form $f \mapsto \hat{f}_{n}$ for some $n \in S$.

\section{REFERENCES}

[1] R.M. Brooks, A ring of analytic functions. Studia Math. 24 (1964) 191-210. MR 30:2363

[2] R.M. Brooks, A ring of analytic functions, II. Studia Math. 39 (1971) 199-208. MR 46:4209

[3] J. Caveny, Bounded Hadamard products of $H^{p}$-functions. Duke Math. J. 33 (1966) 389-394. MR 33:1465

[4] H. Goldmann, Uniform Fréchet algebras. North-Holland, Amsterdam 1990. MR 91f:46073

[5] E. Landau, D. Gaier, Darstellung und Begründung einiger neuerer Ergebnisse der Funktionentheorie. Springer Berlin 1986. MR 88d:01046

[6] H. Render, A. Sauer, Algebras of holomorphic functions with Hadamard multiplication. Studia Math. 118 (1996) 77-100. MR 97b:46070

Universität Duisburg, Fachbereich Mathematik, Lotharstr. 65, D-47057 Duisburg, Federal Republic of Germany

E-mail address: render@math.uni-duisburg.de 